\title{
Multiple scattering of polarized light in uniaxial turbid media with arbitrarily oriented linear birefringence
}

Soichi Otsuki 


\title{
Multiple scattering of polarized light in uniaxial turbid media with arbitrarily oriented linear birefringence
}

\author{
Soichi Otsuki* \\ National Institute of Advanced Industrial Science and Technology (AIST), Health Research Institute, 2217-14, Hayashi-Machi, Takamatsu, \\ Kagawa 761-0395, Japan
}

\begin{abstract}
The effective scattering Mueller matrices obtained by the simulation were simplified to the reduced matrices and factorized using the Lu-Chipman polar decomposition, which afforded the polarization parameters in two dimensions. In general, the scalar retardance around the illumination point of a pencil beam shows a broad azimuthal dependence with an offset. Photons may behave quite differently under the birefringence according to their polarization state. In contrast, when the birefringence is oriented along the $y$-axis in the plane parallel to the surface $(x-y)$ plane, for example, the azimuthal dependence of the scalar retardance shows clear maxima along the $x$ - and $y$-axes and sharp valleys between the maxima. Photons propagating in the medium probably experience the retardance in nearly the same way when they are polarized linearly and circularly. Moreover, the polarization parameters generally become nonsymmetric with respect to the plane perpendicular to both the $x-y$ plane and the plane containing the birefringence axis, which suggests that the pathway of the lateral propagation of photons from the illumination point to the surrounding is slightly oblique upward relative to the $x-y$ plane. These results were also compared with the case in which the birefringence axis is perpendicular to the $x-y$ plane. ๑) 2016 Society of Photo-Optical Instrumentation Engineers (SPIE) [DOI: 10.1117/1.JBO.22.1.015001]
\end{abstract}

Keywords: multiple scattering; backscattering; polarization; birefringence; light propagation in tissues; Monte Carlo simulation. Paper 160580R received Aug. 25, 2016; accepted for publication Dec. 6, 2016; published online Jan. 2, 2017.

\section{Introduction}

Polarization-sensitive optical methods have recently attracted increasing attention for extracting morphological and functional information on biological tissues. ${ }^{1,2}$ Anisotropic organization of many tissues manifests different refractive indices along different directions, yielding linear birefringence. Tissue birefringence can be used as a potentially sensitive probe of the tissue state since anisotropy changes stemming from disease progression or treatment response alter the birefringence properties of the tissue. Several groups have shown that the tissue birefringence certainly provides information for diagnosis of the infarction of heart tissues ${ }^{3}$ and differentiation of healthy and cancerous tissues. ${ }^{4,5}$ Moreover, scalar retardance has been attempted for cancer-suspected zones, as labeled after histological diagnosis. ${ }^{6}$

The quantitative evaluation of the results normally assumes that the fibrous structures of the birefringent tissue lie in a plane parallel to the tissue surface. The optic axis orientation and retardation are extracted using matrix decompositions or numerical procedures by modeling the tissue as a linear retarder. However, this approach is not valid when the fibril orientation is arbitrary. Several theoretical approaches have been described to rigorously analyze the polarimetric effect of anisotropic media with arbitrarily oriented linear birefringence. The differential generalized Jones calculus has been presented, which enables to obtain the differential and Mueller-Jones matrices of such a medium. ${ }^{7}$ This method is based on the generalized $3 \times 3$ Jones calculus ${ }^{8}$ that completely describes the polarization state of an arbitrary three-dimensional light field. A theoretical model of birefringent media has been proposed for biological tissues as a parallel-layered stack with varying optical axis orientations assumed for each layer. ${ }^{9}$ Neither of these theoretical approaches directly treats the depolarization effects, which are essential in turbid media. Some methods have been proposed for analyzing samples with three-dimensional linear birefringence orientation by measuring the polarization-sensitive optical coherence tomography at varying incidence angles ${ }^{10}$ or by imaging a sample twice at different incident angles of the probing beam. ${ }^{11}$ The intrinsic retardance has been determined by utilizing analytical expressions based on the differential generalized Jones calculus. ${ }^{12}$ However, these methods assess only the amplitude and direction of the birefringence but do not fully characterize the polarimetric properties of turbid media.

Meanwhile, the characteristic spatial distributions of the backscattering Mueller matrix of turbid infinite plane media have been investigated based on measurements and Monte Carlo simulations in recent years. ${ }^{13-23}$ We have shown that the socalled reduced effective scattering Mueller matrix, which is transformed from the matrix directly obtained by simulation or measurements, is a complete representation of the polarization nature of turbid media in question. ${ }^{21-24}$ Moreover, the polarization parameters that are extracted using the Lu-Chipman polar decomposition show two-dimensional distributions, which directly express the optical properties of turbid media. Especially, the distribution of the polarization parameters becomes dependent on the azimuth and the radial distance around the illumination point in the presence of linear birefringence. ${ }^{22}$

This paper investigates the polarization parameters of anisotropic turbid infinite plane media with arbitrarily oriented linear birefringence based on a Monte Carlo method, as an extension 
of a previous report. ${ }^{22} \mathrm{We}$ analyze the linear birefringence in turbid media in general for the first time to our knowledge. A framework of a Monte Carlo simulation for birefringent turbid plane media is presented in Sec. 2. The results and discussion are described for anisotropic media with the birefringence axis perpendicular, parallel, and inclined to the plane surface in Secs. 3.1 to 3.3 , respectively.

\section{Monte Carlo Simulation}

The algorithms of the Monte Carlo simulation in this paper are the same as reported elsewhere. ${ }^{22}$ The simulation was performed with the following parameters. The scattering medium had the refractive index of 1.334 , the scattering coefficient of $11.88 \mathrm{~cm}^{-1}$, and a negligible absorption. The birefringence of $1.36 \times 10^{-5}$ existed along an arbitrary direction that is designated for each simulation. The birefringent effect on the polarization is only considered during the paths of light between scattering events in the same manner as in a previous report. ${ }^{22}$ Spherical particles with the radius of $0.7 \mu \mathrm{m}$ and the refractive index of 1.59 were used. The photons' wavelength was $632.8 \mathrm{~nm}$. Hence, the asymmetry parameter was estimated to be 0.932 . The photons were incident on the sample surface in the positive $z$ direction at the same point $(0,0,0)$, implying a zero beam diameter. The acceptance angle for the exiting photons was $90 \mathrm{deg}$. The calculation was repeated until the passage of $5 \times 10^{8}$ photons was simulated. The plane surface was divided into grids per $0.1 \mathrm{~mm}$ and $1 \mathrm{deg}$ in the radial and azimuthal directions, respectively. The stokes vector of a photon exiting from each grid was multiplied by the photon weight, accumulated, and finally divided by the number of total photons.

The effective backscattering Mueller matrix $\tilde{\mathbf{M}}$ obtained by the simulations is generally represented in two dimensions in anisotropic media by ${ }^{22}$

$\tilde{\mathbf{M}}(r, \phi)=\mathbf{R}(-\phi) \tilde{\mathbf{M}}^{r}(r, \phi) \mathbf{R}(-\phi)$,

where $r$ and $\phi$ are the polar coordinates of each point on the surface of the medium, $\tilde{\mathbf{M}}^{r}$ is the reduced scattering matrix, and $\mathbf{R}$ is the rotation matrix as represented by

$\mathbf{R}(\phi)=\left[\begin{array}{cccc}1 & 0 & 0 & 0 \\ 0 & \cos 2 \phi & \sin 2 \phi & 0 \\ 0 & -\sin 2 \phi & \cos 2 \phi & 0 \\ 0 & 0 & 0 & 1\end{array}\right]$.

The reduced matrix could mathematically factorized along the azimuthal or radial direction around the illumination point in one dimension or over the plane surface in two dimensions by the Lu-Chipman polar decomposition into the diattenuator $\mathbf{M}_{\mathrm{D}}$, retarder $\mathbf{M}_{\mathrm{R}}$, and depolarizer $\mathbf{M}_{\Delta}$ matrices. ${ }^{25}$ The polarization parameters were deduced from the decomposed matrices as described previously. ${ }^{22}$ These parameters were: the depolarization coefficients for the horizontally vertically, \pm 45 deg linearly, and circularly polarized light $\left(\alpha_{1}, \alpha_{2}\right.$, and $\alpha_{3}$, respectively); the horizontal, 45 deg linear, and circular diattenuations $\left(D_{\mathrm{H}}, D_{45}\right.$, and $D_{\mathrm{C}}$, respectively); the linear retardance $\delta$; the optical rotation $\psi$; and the orientation of the linear retardation $\theta$.

When the polarization parameters were examined as a function of the azimuth or the radial distance around the illumination point, the elements of the reduced matrix at each grid were calculated before the matrix decomposition by averaging the values of the surrounding grids to reduce noise in the variations with the azimuth or the distance, in the same manner as the previous report. ${ }^{22}$

\section{Results and Discussion}

\subsection{Birefringence Axis Perpendicular to the Plane Surface}

Multiple scattering of polarized light was simulated for an anisotropic infinite plane medium with birefringence axis oriented vertical to the plane surface. The reduced effective scattering matrix was hardly dependent on the azimuth around the illumination point but only dependent on the radial distance. Hence, the matrix elements were azimuthally averaged at varying radial distances. The averaged matrix elements are shown as a function of the radial distance in Fig. 1. Small but considerable values are found for $m_{02}, m_{12}, m_{13}, m_{20}, m_{21}$, and $m_{31}$. These elements exactly satisfy reciprocity; $m_{20}=-m_{02} ; m_{21}=-m_{12} ; m_{31}=$ $m_{13}$. Some elements nearly satisfy reciprocity; $m_{10} \approx m_{01}$; $m_{32} \approx-m_{23}$. The simulation was also conducted for the acceptance angle of $10 \mathrm{deg}$ for exiting photons and the total photon number of $10^{9}$ with the other conditions being kept the same. It was found that the reduced matrix exactly fulfills reciprocity in this case, namely $m_{10}=m_{01}$ and $m_{32}=-m_{23}$ as well as the other nondiagonal elements. The exact reciprocity may require that the direction of beam exit from the infinite plane medium is approximately normal to the plane surface.

The reduced matrix averaged azimuthally was decomposed to provide the polarization parameters as a function of the radial distance, as shown in Fig. 2. Note that the $|\delta|$ value becomes approximately zero for distances in the 0.5 to $2.2-\mathrm{mm}$ range. Meanwhile, $\alpha_{2}$ and $\alpha_{3}$ values exhibit jumps at distances of



Fig. 1 Elements of the reduced effective scattering Mueller matrix as a function of the radial distance for anisotropic media with birefringence axis perpendicular to the plane surface. The matrix elements as the ordinates are normalized by $m_{00}$ and dimensionless. 

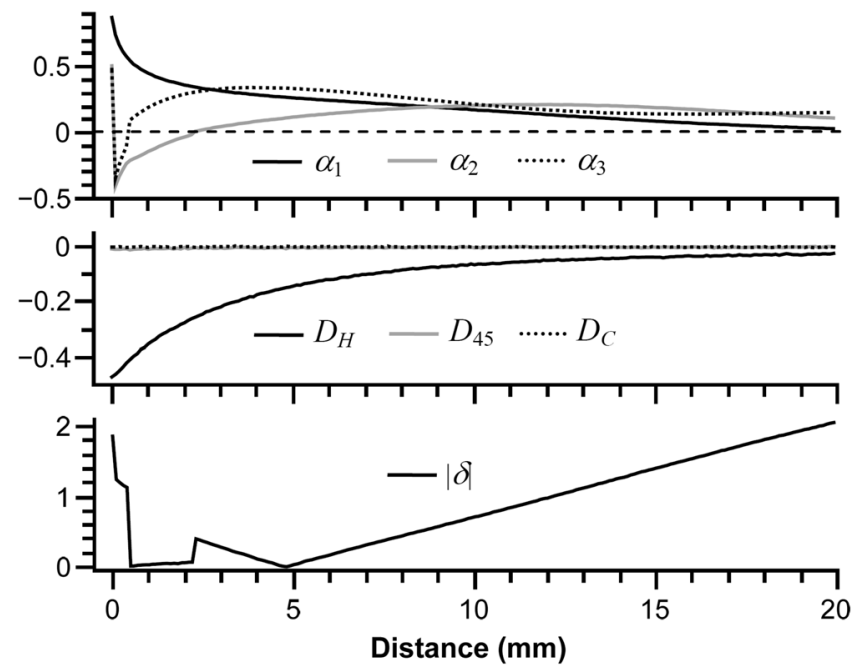

Fig. 2 Polarization parameters as a function of the radial distance for anisotropic media with birefringence axis perpendicular to the plane surface. The ordinates are dimensionless except $|\delta|$ whose unit is radian.

0.5 and $2.2 \mathrm{~mm}$. These facts indicate that the decomposition fails in the 0.5 to $2.2-\mathrm{mm}$ range, whereas it yields physically reasonable matrix factors for distances longer than $2.2 \mathrm{~mm}$, similar to the case of isotropic infinite plane media. ${ }^{21}$ Such a trend was also seen for the anisotropic media with the birefringence axis parallel or inclined to the plane surface, which are described in the succeeding sections.

The $\alpha_{3}$ value is reduced by 0.3 at maximum in the distance range longer than $10 \mathrm{~mm}$ relative to the case for the isotropic media. ${ }^{21}$ The $\alpha_{1}$ value is also reduced by 0.1 at maximum, whereas the $\alpha_{2}$ value barely changes in the same distance range, which results in $\alpha_{1}$ being smaller than the $\alpha_{2}$ value. The two coefficients are nearly the same in the long distance range due to randomization of linear polarizations for isotropic media. It is likely that the greater depolarization for the horizontally and circularly polarized light comes from selective depolarization due to the birefringence perpendicular to the plane surface.

The $D_{\mathrm{H}}$ value displays negative value that decreases with decreasing the distance, whereas $D_{45}$ and $D_{\mathrm{C}}$ have negligible values over the whole distance. The $D_{\mathrm{H}}$ value is -0.26 at the distance of $2.2 \mathrm{~mm}$, which is close to the value for an isotropic medium. ${ }^{21}$ Probably, the horizontal linear diattenuation arising from the nature of the single scattering increases with the distance approaching the illumination point.

A double-scattering model has been proposed to rationalize the simulation results for a birefringent infinite plane medium. ${ }^{22}$ Briefly, although the photon propagates along many possible curved trajectories, photon trajectories may be approximated by three straight lines from the entrance $O$ to the exit $P$ via the intermediate points $A$ and $B$, as seen in Fig. 3(a). It is likely that the direction of the electric vector of the photon wave as well as the photon's local reference frame is fairly preserved after repeated scatterings close to the forward direction, as seen in Fig. 3(b).

According to the double-scattering model, the vertically propagating photon does not produce the retardation because the propagation direction is parallel to the birefringence axis. On the other hand, linearly polarized photons propagate laterally in the birefringent medium as shown in Fig. 4. Apparently, the $\delta$
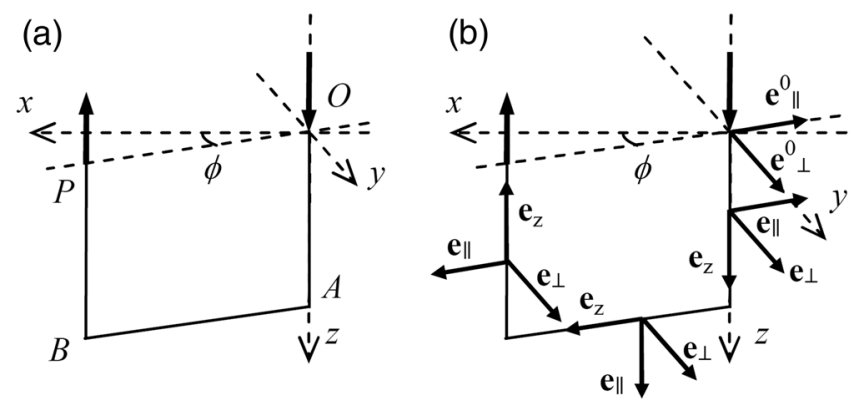

Fig. 3 Double scattering model. (a) Approximation of the photon trajectory by three lines. (b) Configuration of the local reference frame during the photon propagation.



Fig. 4 Local reference frame and the polarization orientation during the lateral propagation of a polarized photon.

value is positive during the lateral propagation of the photon because the horizontal axis of the local reference frame of the photon $\mathbf{e}_{\|}$is parallel to the birefringence axis. In the mean time, a left-handed circular polarization of a photon is converted to the left-handed elliptical polarization with the major axis orientation $\mathbf{E}$ inclined at $45 \mathrm{deg}$ to the birefringence axis, as seen in Fig. 4. Evidently, the positive value for $\delta$ is also generated. Then let us examine the variation of $|\delta|$ with the distance in Fig. 2. The $|\delta|$ value decreases to zero and increases up to $\sim 2.1$ when increasing the distance. This variation indicates that the $\delta$ value increases from a negative value to a positive value. The negative value at distances shorter than $4.7 \mathrm{~mm}$ is of the linear retardance, which may reflect from the nature of the single scattering, and decreases with increasing the distance, as observed for an isotropic medium. ${ }^{21}$ The positive value at distances longer than $4.7 \mathrm{~mm}$ is of the retardance, which comes from the medium birefringence, and almost linearly increases with the distance. The slope of the linear variation of $|\delta|$ is $1.35 \mathrm{~cm}^{-1}$, which is lower than the value, $1.64 \mathrm{~cm}^{-1}$, as estimated in Sec. 3.2, for the anisotropic medium with the birefringence axis parallel to the plane surface and the same birefringence amplitude. This is probably because the vertical propagation does not contribute to the retardation for the case in which the birefringence axis is perpendicular to the plane surface.

\subsection{Birefringence Axis Parallel to the Plane Surface}

Multiple scattering of polarized light has been described in detail for an anisotropic infinite plane medium with birefringence axis 




Fig. 5 Absolute values of the retardance $|\delta|$ as a function of the azimuth for anisotropic media with birefringence of $0.68 \times 10^{-5}$ (solid), $1.36 \times 10^{-5}$ (gray), and $2.72 \times 10^{-5}$ (dotted).

oriented parallel to the plane surface. ${ }^{22}$ In this study, simulations were performed for anisotropic media that have the same birefringence orientation but have different birefringence amplitudes. Because the reduced effective scattering matrices were dependent on the azimuth and the radial distance around the illumination point, the matrices were factorized in two dimensions to obtain the polarization parameters. The retardations for these media are shown, as a function of the azimuth at the radial distance of $5 \mathrm{~mm}$, in Fig. 5. The $|\delta|$ value shows periodic changes with the azimuth; it displays two larger maxima at 0 deg and $180 \mathrm{deg}$ and two smaller maxima at $90 \mathrm{deg}$ and $270 \mathrm{deg}$ for all media. The retardations are also shown along the radial distance at the azimuth of 0 deg in Fig. 6 . For the birefringence of $0.68 \times 10^{-5}$, the $|\delta|$ value continuously increases almost linearly up to $\sim 2.0$ with distance except for the range smaller than $2.4 \mathrm{~mm}$, where the polarization parameters are not realistic, as noted in Sec. 3.1. In contrast, after the $|\delta|$ value increases to $\pi$, it turns into a decrease for the two cases with larger values of the birefringence. The retardance $\delta$ is negative and increases in its absolute value with the distance at the azimuth of $0 \mathrm{deg}$, as described previously. ${ }^{22}$ Hence, the $\delta$ value decreases almost linearly in the distance range longer than $\sim 3 \mathrm{~mm}$ for the two larger values of birefringence. The corresponding slopes of the linear variation are $-0.641,-1.64$, and $-3.23 \mathrm{~cm}^{-1}$ in the distance range of 10 to $20 \mathrm{~mm}$ for the birefringence values of $0.68 \times 10^{-5}, 1.36 \times 10^{-5}$, and $2.72 \times 10^{-5}$, respectively. Thus, the slope for the $\delta$ variation becomes greater nearly in proportion to the birefringence value at the azimuth of $0 \mathrm{deg}$.

When the birefringence slow axis is parallel to the plane surface, the retardation $\delta$ exhibits characteristic variations with the azimuth $(\phi)$ depending on whether the photon propagates laterally or vertically, as shown in Fig. $7 .^{22}$ When the slow axis is



Fig. 6 Absolute values of the retardance $|\delta|$ along the radial distance. See the caption to Fig. 5.

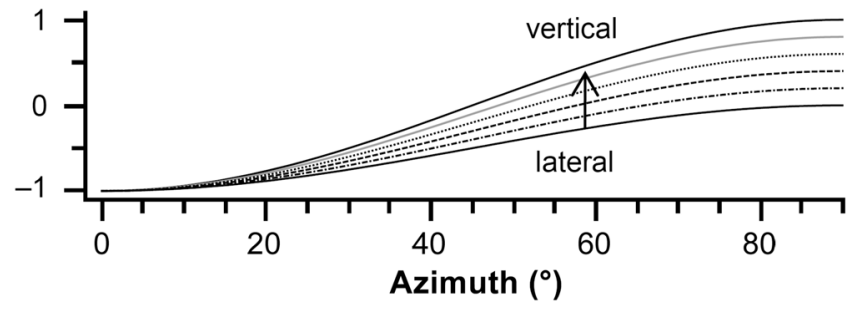

Fig. 7 Azimuthal dependence of the relative retardation produced during the lateral and vertical propagations of light. The ordinate unit is arbitrary. The two main contributions are mixed with varying their ratio, which produces intermediate dependences.

along the $y$ axis, the $\delta$ value becomes the largest negative along the $x$ axis $(\phi=0 \mathrm{deg})$ and increases up to 0 with $\phi$ for the laterally propagating photon. Meanwhile, it is also the largest negative along the $x$ axis, increases with increasing $\phi$, and becomes the largest positive along the $y$ axis $(\phi=90 \mathrm{deg})$ for the vertically propagating photon. This may be true for linearly and circularly polarized photons. It is also assumed that photons propagating laterally and vertically independently contribute to the $\delta$ value. It is likely that photons with the horizontalvertical polarization generate no retardation and that the $|\delta|$ value becomes larger, as the orientation of polarization approaches $\pm 45 \mathrm{deg}$ from $0 \mathrm{deg}$ or $90 \mathrm{deg}$. On the other hand, the circularly polarized photons may be transformed by the birefringence to the elliptical polarized photons with the orientation of the major axis at \pm 45 deg during both lateral and vertical propagations. It is suggested that the two main dependences due to the lateral and vertical propagations of photons are dominant and that the actual variations of $\delta$ are made from mixing of the two dependences. These assumptions are required to generate the characteristic azimuthal variations of the $|\delta|$ value, as shown in Fig. 5; the $|\delta|$ value takes larger maxima at $0 \mathrm{deg}$ and $180 \mathrm{deg}$ (largest negative $\delta$ ) and takes smaller maxima at $90 \mathrm{deg}$ and $270 \mathrm{deg}$ (largest positive $\delta$ ).

\subsection{Birefringence Axis Inclined to the Plane Surface}

Multiple scattering of polarized light is simulated for anisotropic media with the birefringence axis that is inclined at $45 \mathrm{deg}$ to the plane surface along the $y$ axis. The polarization parameters are shown as two-dimensional maps in Fig. 8 (the matrix elements were not averaged). Each image shows a circular $2 \mathrm{~cm}$ radius area centered at the point of beam incidence. The parameters are symmetric or antisymmetric with respect to the $y-z$ plane but are not either with respect to the $x-z$ plane. In contrast, the parameters show second- or fourth-order rotational symmetry when the birefringence axis is parallel to the plane surface. ${ }^{22}$ This symmetric feature is remarkable for the $\alpha_{2}$ value, as seen in the two-dimensional maps of Fig. 9, for media with the birefringence axis inclined at varying angles to the plane surface. The nonsymmetry with respect to the $x-z$ plane is obvious even for the inclination angle of only $9.9 \mathrm{deg}$. Moreover, $\alpha_{2}$ is larger along the $x$ axis than along the $y$ axis for the birefringence parallel to the plane surface, whereas the $\alpha_{2}$ variation becomes more prominent between the positive and the negative $y$ directions as the inclination angle increases.

The parameters are also shown as a function of the azimuth around the illumination point at the radial distance of $15 \mathrm{~mm}$ in Fig. 10. Evidently, $D_{\mathrm{H}},|\delta|$, and $\theta$ are nonsymmetric between the two ranges of $0 \mathrm{deg}$ to $180 \mathrm{deg}$ and $180 \mathrm{deg}$ to $360 \mathrm{deg}$. Then, let 

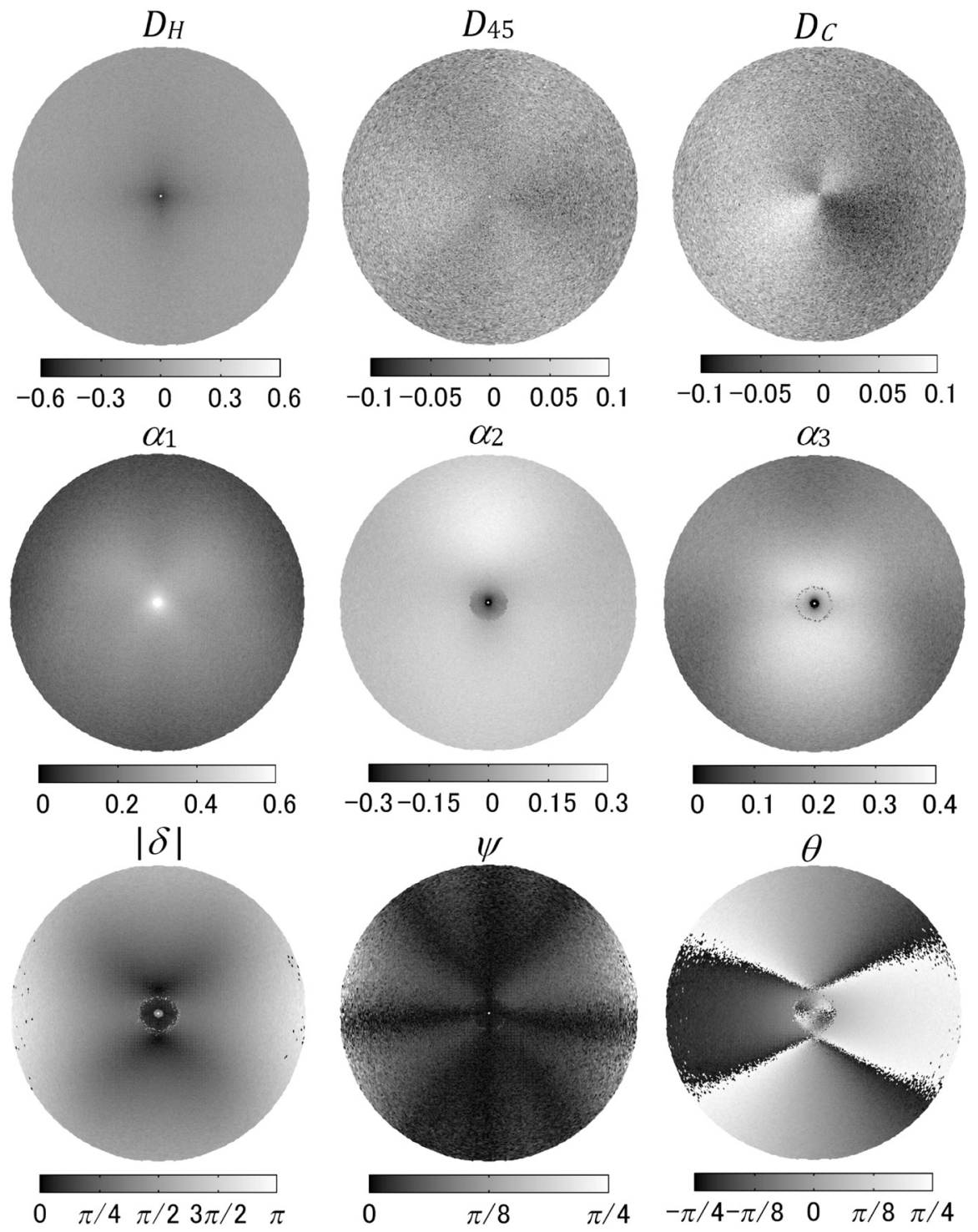

Fig. 8 Two-dimensional maps of the polarization characteristics. The scale bars are dimensionless except $|\delta|,|\psi|$, and $\theta$ whose unit is radian.

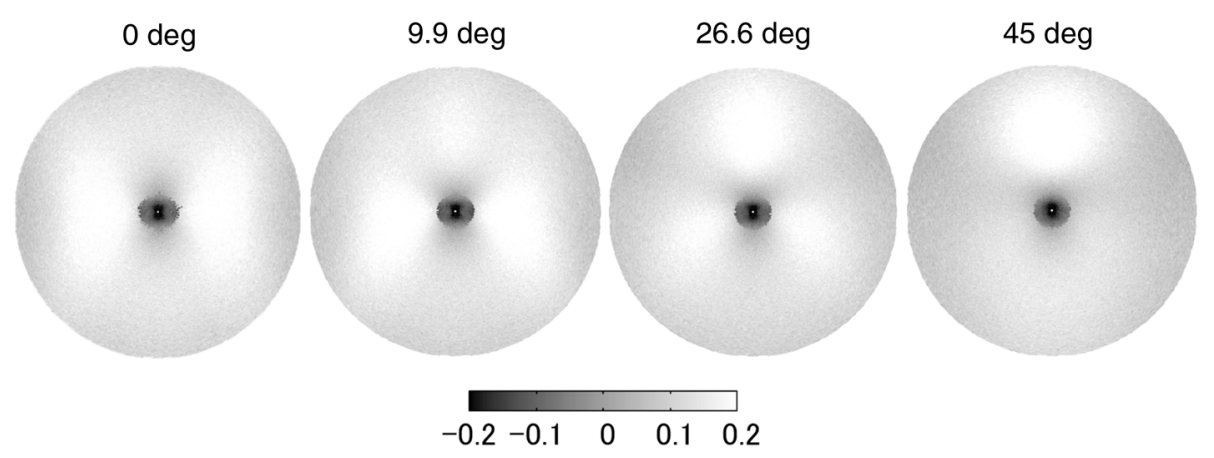

Fig. 9 Depolarization coefficient for the 45 deg linearly polarized light $\alpha_{2}$ for media with the birefringence axis inclined at the designated angles. The scale bar is dimensionless.

us pay attention to the dependence of $|\delta|$. There are two large maxima at the azimuths of $0 \mathrm{deg}$ and $180 \mathrm{deg}$ and two small minima at the azimuths of $90 \mathrm{deg}$ and $270 \mathrm{deg}$. The $|\delta|$ value shows shallow valleys between these maxima, of which the bottom has offset values of $\sim 1.2$. This is also in contrast to the case in which the birefringence axis is parallel to the plane surface.

Let us examine using the double-scattering model how the birefringence affects the retardance according to the propagation direction of photons. When a photon propagates vertically to the 

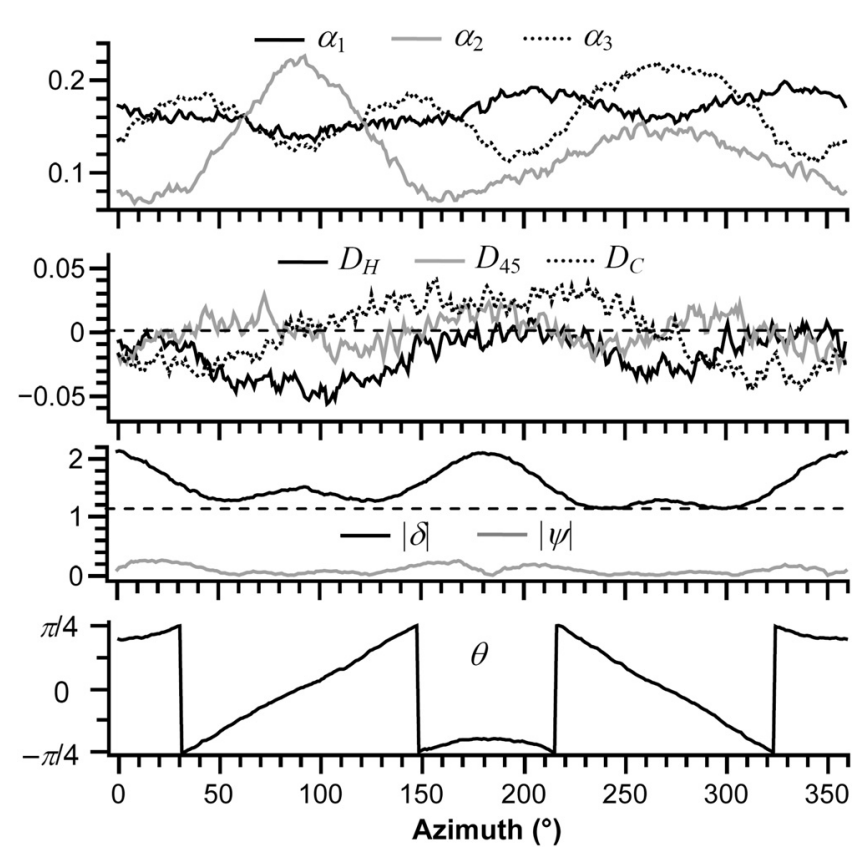

Fig. 10 Polarization characteristics along the azimuthal direction at 15-mm distance. The ordinates are dimensionless except $|\delta|,|\psi|$, and $\theta$ whose unit is radian.

surface plane, it propagates forward at the origin into the page and, after it travels laterally along the azimuth $\phi$, it propagates backward out of the page, as seen in Fig. 11(a). The local reference frame $\mathbf{e}_{\|}$and $\mathbf{e}_{\perp}$ is aligned along the azimuth for the forward and backward propagations; the horizontal axis $\mathbf{e}_{\|}$becomes reversed in direction between both propagations. Because the birefringence slow axis for the photon $\mathbf{b}^{\prime}$ is along the $y$ axis, the photon may behave in the same manner as the case in which the slow axis itself is along the $y$ axis. Next, examine a photon propagating laterally along the surface plane. Assume that the birefringence slow axis $\mathbf{b}$ is inclined at $45 \mathrm{deg}$ to the surface plane. When the horizontal axis of the laboratory reference frame $\mathbf{e}_{\|}^{0}$ is aligned along the $x$ axis $(\phi=0 \mathrm{deg})$, as seen in Fig. 11(b), the birefringence slow axis $\mathbf{b}$ itself is parallel to the local reference frame $\mathbf{e}_{\perp}$ and $\mathbf{e}_{\|}$and is inclined at an orientation $\theta$


Fig. 11 Configuration of the local reference frame when a photon propagates (a) vertically, and laterally along (b) the $x$ axis and (c) an azimuth $\phi$.

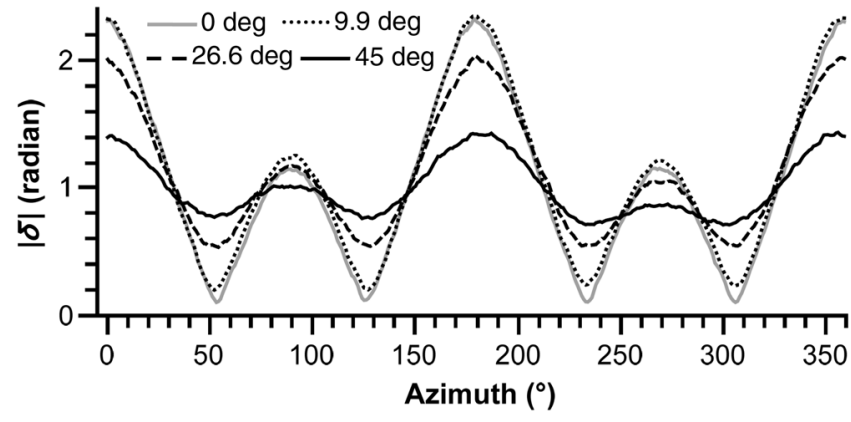

Fig. 12 Absolute values of the retardance $|\delta|$ along the azimuthal direction at the 10-mm distance. The birefringence axis is inclined at the designated angles.

of $45 \mathrm{deg}$ to the horizontal axis $\mathbf{e}_{\|}$. As the axis $\mathbf{e}_{\|}^{0}$ makes an azimuth $\phi$ to the $x$ axis, the slow axis for photon $\mathbf{b}^{\prime}$ is oriented at an angle smaller than $45 \mathrm{deg}$ to the axis $\mathbf{e}_{\|}$, as seen in Fig. 11(c). Because the orientation of the slow axis for the photon changes with the azimuth, the linearly polarized photon behaves differently depending on its orientation of polarization. In addition, the circularly polarized photon is converted to the elliptically polarized photon with different orientations of the major axis depending on the azimuth.

The retardation $\delta$ is shown as a function of the azimuth for the birefringence whose axis is inclined at varying angles to the plane surface in Fig. 12. As the inclination angle becomes larger, the amplitudes of the maxima decrease, the valleys between the maxima are broadened, and the offset values become larger. This clearly reveals that, the more inclined the birefringence axis, photons propagating laterally produce a more complex mixture of the scalar and vector retardances, which leads to less characteristic dependence of the $\delta$ value on the azimuth. Although the vertical propagation still keeps its characteristic feature in the $\delta$ value, its contribution is more reduced. Thus, the total contribution from the lateral and vertical propagations to the $\delta$ value shows a broader variation with a larger offset.

Next, let us inspect the azimuthal dependence of the orientation of the fast axis $\theta$ as a linear retarder of the birefringent medium, as seen in Fig. 13. For the birefringence with its axis parallel to the plane surface, as the azimuth increases from $0 \mathrm{deg}$ to $90 \mathrm{deg}, \theta$ decreases from 0 to $-\pi / 4$, turns its sign at an intermediate azimuth, and decreases to 0 . This azimuthal dependence corresponds to the orientation of the fast axis relative to the horizontal axis of the laboratory reference frame $\mathbf{e}_{\|}^{0}$; the $\theta$ change corresponds to the orientation change from $0 \mathrm{deg}$ to $-45 \mathrm{deg}$

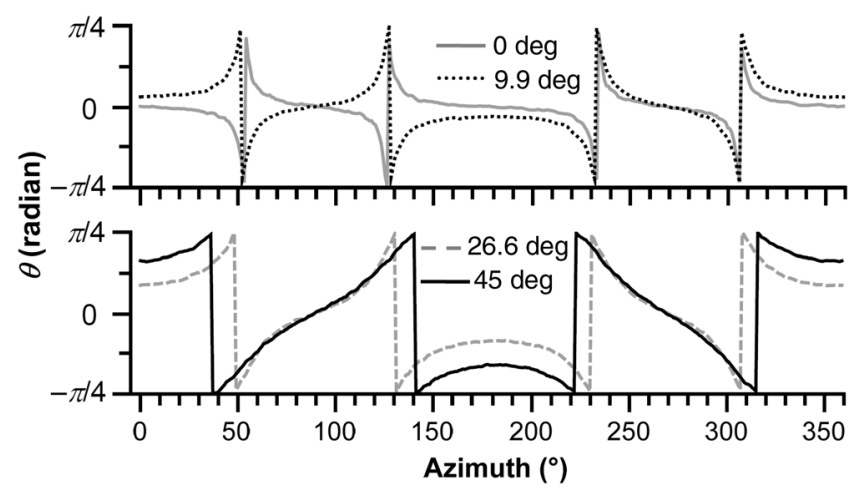

Fig. 13 Fast-axis orientation as a linear retarder along the azimuthal direction at the $10-\mathrm{mm}$ distance. The birefringence axis is inclined at the designated angles. 
below the intermediate azimuth and from $-45 \mathrm{deg}$ to $-90 \mathrm{deg}$ above the intermediate azimuth. Because the orientation of the fast axis is constant at 0 deg during the lateral propagation of photons, it is mainly determined by the vertical propagation. However, when the birefringence is inclined at $9.9 \mathrm{deg}, \theta$ has a value $\sim 0.1$ at the azimuth of $0 \mathrm{deg}$. As the azimuth increases to $90 \mathrm{deg}$, the orientation $\theta$ increases to $\pi / 4$ until an intermediate azimuth, turns its sign, and then increases to 0 . This azimuthal dependence is partly influenced by the lateral propagation; the $\theta$ change corresponds to the orientation change of the fast axis from $-9.9 \mathrm{deg}$ to $-45 \mathrm{deg}$ below the intermediate azimuth and from -45 deg to $-90 \mathrm{deg}$ above the intermediate azimuth. Note that the sign of $\theta$ in the range of 0 deg- 180 deg is reversed between the cases in which the birefringence axis is parallel and inclined to the plane surface. This may originate from the different orientation changes of the fast axis during the lateral and vertical propagations of photons. The orientation turns its sign around the azimuths of $0 \mathrm{deg}$ and $180 \mathrm{deg}$ during the vertical propagation, whereas it does not during the lateral propagation. Even though the inclination of the birefringence increases to $45 \mathrm{deg}$, the fundamental variation of $\theta$ is the same; the value at the azimuth 0 deg increases and the smallest intermediate azimuth shifts to a smaller azimuth.

Finally, let us examine why the polarization parameters are symmetric or antisymmetric with respect to the $y-z$ plane, but why they are not either with respect to the $x-z$ plane for anisotropic media with birefringence directions that are inclined to the surface plane. The distributions of the polarization parameters are different due to the nonsymmetry below and above the $x$ axis for only a $9.9 \mathrm{deg}$ inclination of the birefringence direction, as noted above. This suggests that the nonsymmetry does not come from the birefringence itself but comes from the nature

(a)


(1)

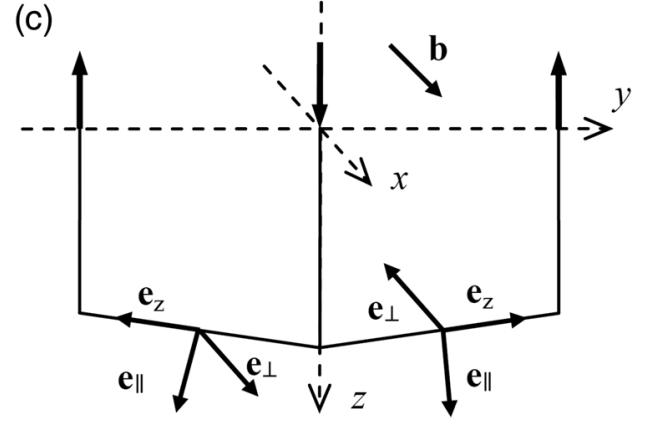

Fig. 14 Modification of the double-scattering model in which the lateral propagation direction is oblique at a small angle $\chi$ with respect to the plane surface. (a) A photon trajectory, (b) its approximation by three lines, and (c) the configuration of the local reference frame during the photon propagation. of the multiple scattering in infinite plane media. A possible explanation is as follows. The photon that is injected normal to the plane surface vertically travels into the medium up to a fairly deep depth due to the first vertical propagation and the following near-forward scattering events. Then the photon migrates away from the central area to the radial distance, as shown in Fig. 14(a). Such a photon trajectory may be well approximated by a double-scattering model in which the lateral propagation after the first scattering is oblique upward at a small angle $\chi$ with respect to the plane surface, as is represented by a line $\mathrm{AB}$ in Fig. 14(b). This obliqueness is originated from the nature of the multiple scattering but is not influenced by the direction and amplitude of the birefringence. The effective birefringence for the propagating photon becomes larger in the positive $y$ direction than in the negative $y$ direction due to the oblique lateral propagation because the angle between the birefringence and the propagation direction of the photon is closer to 90 deg along the positive $y$ direction, as seen in Fig. 14(c). Apparently, the effective birefringence is identical between the positive and negative directions along the $x$ axis in spite of the oblique propagation. Thus, the difference in the effective birefringence causes the nonsymmetry of the polarization parameters with respect to the $x-z$ plane in anisotropic infinite plane media with the birefringence direction that is inclined to the plane surface. It is likely that the two-dimensional distribution of the polarization parameters is not affected very much for media whose birefringence axis is parallel to the plane surface, when the lateral propagation is oblique at angles smaller than $\sim 10 \mathrm{deg}$. This is supported by the fact that the $|\delta|$ variation for the case in which the birefringence axis is inclined at 9.9 deg to the plane surface is not very different from the case of zero inclination of the birefringence axis, as seen in Fig. 12.

\section{Conclusions}

We presented polarimetric analyses of backward scattering from uniaxial turbid media with arbitrarily oriented birefringence using a Monte Carlo method. Our approach takes advantage of the reduced effective scattering Mueller matrix, which completely and precisely expresses the optical response of turbid infinite plane media to a polarized point source. The polarization parameters were obtained using the Lu-Chipman decomposition of the reduced matrix in one dimension along the azimuthal or radial direction or in two dimensions over the plane surface. When the birefringence axis is oriented parallel to the $x-y$ plane, the scalar retardance around the illumination point takes clear maxima and sharp valleys between the maxima, of which bottoms attain virtually zero. On the basis of the double-scattering model, this azimuthal dependence probably indicates that photons propagating in the medium experience the retardance in nearly the same way when they are polarized linearly and circularly and that the circularly polarized photons are transformed by the birefringence to the elliptical polarized photons with the major axis orientated at $\pm 45 \mathrm{deg}$. In contrast, when the birefringence axis is inclined to the $x-y$ plane, the azimuthal dependence of the scalar retardance becomes broader with an offset. It is likely that photons behave quite differently under the birefringence according to their polarization state and that the circular polarizations are converted to the elliptical polarizations with different major axis orientations depending on the propagation azimuth. Moreover, when the inclined birefringence axis is along the $y$ axis, the polarization parameters become nonsymmetric with respect to the $x-z$ plane, which implies that the 
lateral pathway of photons from the illumination point to the radial distance is slightly oblique upward relative to the $x-y$ plane. In addition, when the birefringence axis is perpendicular to the $x-y$ plane, the reduced effective scattering Mueller matrix has little azimuthal dependence so that the matrix elements were azimuthally averaged at varying radial distances. It was found that the retardance is negative in the short distance range, which reflects from the nature of the single scattering, and that it increases with distance and becomes positive in the middle and large distance range due to the medium birefringence.

The present work solved how the linear birefringence appears as the scalar retardance and influences the other polarization parameters in turbid infinite plane media in a general way for the first time to our knowledge. The numerical results presented here should contribute to the understanding of the essential physical processes of polarized light propagation in birefringent biological tissues and provide potential methods for use in actual medical diagnosis.

\section{Disclosures}

The author has no other relevant financial interests in the manuscript than that stated in Acknowledgment and no other potential conflicts of interest to disclose.

\section{Acknowledgments}

This work was supported by Japan Society for the Promotion of Science (JSPS) KAKENHI Grant Number JP26390092.

\section{References}

1. N. Ghosh and I. A. Vitkin, "Tissue polarimetry: concepts, challenges, applications, and outlook," J. Biomed. Opt. 16, 110801 (2011).

2. S. Alali and I. A. Vitkin, "Polarized light imaging in biomedicine: emerging Mueller matrix methodologies for bulk tissue assessment," J. Biomed. Opt. 20, 061104 (2015).

3. M. F. G. Wood et al., "Polarization birefringence measurements for characterizing the myocardium, including healthy, infarcted and stem-cell-regenerated tissues," J. Biomed. Opt. 15, 047009 (2010).

4. A. Pierangelo et al., "Polarimetric imaging of uterine cervix: a case study," Opt. Express 21, 14120-14130 (2013).

5. M. Sun et al., "Characterizing the microstructures of biological tissues using Mueller matrix and transformed polarization parameters," Biomed. Opt. Express 5, 4223-4234 (2014).

6. J. Rehbinder et al., "Ex vivo Mueller polarimetric imaging of the uterine cervix: a first statistical evaluation," J. Biomed. Opt. 21, 071113 (2016).

7. N. Ortega-Quijano and J. L. Arce-Diego, "Generalized Jones matrices for anisotropic media," Opt. Express 21, 6895-6900 (2013).

8. R. M. A. Azzam, "Three-dimensional polarization states of monochromatic light fields," J. Opt. Soc. Am. A 28, 2279-2283 (2011).
9. F. Fanjul-Vélez and J. L. Arce-Diego, "Polarimetry of birefringent biological tissues with arbitrary fibril orientation and variable incidence angle," Opt. Lett. 35, 1163-1165 (2010).

10. N. Ugryumova, S. V. Gangnus, and S. J. Matcher, "Three-dimensional optic axis determination using variable-incidence-angle polarizationoptical coherence tomography," Opt. Lett. 31, 2305-2307 (2006).

11. M. A. Wallenburg et al., "Polarimetry-based method to extract geometry-independent metrics of tissue anisotropy," Opt. Lett. 35, 2570-2572 (2010).

12. N. Ortega-Quijano, F. Fanjul-Vélez, and J. L. Arce-Diego, "Polarimetric study of birefringent turbid media with three-dimensional optic axis orientation," Biomed. Opt. Express 5, 287-292 (2014).

13. A. H. Hielscher et al., "Diffuse backscattering Mueller matrices of highly scattering media," Opt. Express 1, 441-454 (1997).

14. M. J. Raković et al., "Light backscattering polarization patterns from turbid media: theory and experiment," Appl. Opt. 38, 3399-3408 (1999).

15. S. Bartel and A. H. Hielscher, "Monte Carlo simulations of the diffuse backscattering Mueller matrix for highly scattering media," Appl. Opt. 39, 1580-1588 (2000).

16. X. Wang, G. Yao, and L. H. Wang, "Monte Carlo model and single scattering approximation of the propagation of polarized light in turbid media containing glucose," Appl. Opt. 41, 792-801 (2002).

17. X. D. Wang and L. V. Wang, "Propagation of polarized light in birefringent turbid media: a Monte Carlo study," J. Biomed. Opt. 7, 279-290 (2002).

18. P. Yang et al., "Sensitivity of the backscattering Mueller matrix to particle shape and thermodynamic phase," Appl. Opt. 42, 4389-4395 (2003).

19. J. Dillet et al., "Size determination by use of two-dimensional Mueller matrices backscattered by optically thick random media," Appl. Opt. 45, 4669-4678 (2006).

20. C. Baravian, J. Dillet, and J. P. Decruppe, "Birefringence determination in turbid media," Phys. Rev. E 75, 032501 (2007).

21. S. Otsuki, "Multiple scattering of polarized light in turbid infinite planes: Monte Carlo simulations," J. Opt. Soc. Am. A 33, 988-996 (2016).

22. S. Otsuki, "Multiple scattering of polarized light in turbid birefringent media: a Monte Carlo simulation," Appl. Opt. 55, 5652-5664 (2016).

23. S. Otsuki, "Multiple scattering in turbid media containing chiral components: a Monte Carlo simulation," Opt. Commun. 382, 157-161 (2017).

24. S. Otsuki, "Symmetry relationships for multiple scattering of polarized light in turbid spherical samples: theory and a Monte Carlo simulation," J. Opt. Soc. Am. A 33, 258-269 (2016).

25. S. Y. Lu and R. A. Chipman, "Interpretation of Mueller matrices based on polar decomposition," J. Opt. Soc. Am. A 13, 1106-1113 (1996).

Soichi Otsuki received his master's and PhD degrees in applied chemistry from Osaka City University, Japan. He is now a research manager of the National Institute of Advanced Industrial Science and Technology, Japan. Currently, his major research interests lie in ellipsometry and polarimetry, interaction between light and complex turbid medium like biological tissue, and biomedical imaging. 